\title{
DiaMap: New applications for processing IR spectra of fluid-rich diamonds and mapping diamonds containing isolated nitrogen (Type Ib) and boron (Type IIb)
}

\author{
Daniel Howell ${ }^{1}$, Yaakov Weiss ${ }^{2}$, Karen V. Smit ${ }^{3}$, Lorne Loudin ${ }^{3}$, Fabrizio Nestola ${ }^{1}$ \\ ${ }^{1}$ Universitàdi Padova, I-35131 Padova, Italy.dan@ddl.diamonds,fabrizio.nestola@unipd.it \\ ${ }^{2}$ Lamont-Doherty Earth Observatory of Columbia University, Palisades, NY, USA.yweiss@ldeo.columbia.edu \\ ${ }^{3}$ Gemological Institute of America, New York City, NY, USA. ksmit@gia.edu, lloudin@gia.edu
}

\section{Introduction}

Since infrared (IR) spectroscopy was first used to characterise diamonds in the 1930's (Robertson et al., 1934), it has become a commonly used, non-destructive tool in diamond research. Not only does it provide information on various nitrogen-related defects, it can also be used to characterize fluid micro-inclusions in diamonds (Navon et al., 1988), providing key information on different C-O-Hbearing mantle fluid types, and water and carbonate cycling in the Earth's deep interior.

IR studies of diamonds are typically limited to single point analyses, while linear transects of multiple points have also been used to show core to rim variations in nitrogen content and aggregation state, and fluid micro-inclusions composition. Technological developments over the past 15 years, however, have made the application of IR-mapping feasible. DiaMap freeware was developed to take advantage of these new applications (Howell et al., 2012). It allows automated and easy processing of the abundant IR spectra obtained from the IR-mapping of a single diamond, and provides spatial quantitative information of nitrogen- and hydrogen-related defects. IR-mapping of cuboid and fibrous diamonds showed that our traditional understanding from monocrystaline diamonds on the nitrogen aggregation rates and platelet development does not apply to all diamond growth types (Howell et al., 2012). Moreover, since nitrogen aggregation in diamonds is temperature and time dependent, diamond IR-mapping is advantageous for understanding the geological history of natural diamond. Here we report new applications of DiaMap software, allowing easy and quantitative determination of the mineralogy and type of fluids in micro-inclusion-bearing diamonds (DiaMap_Fluid) and automated deconvolution of IR spectral maps collected across plates of Type Ib and IIb diamonds (DiaMap_Ib and IIb).

\section{DiaMap_Fluid}

High-density fluids (HDFs) encapsulated in micro-inclusions within diamonds vary between four major compositional types: saline, silicic, and high-Mg and low-Mg carbonatitic. IR spectroscopy is the only method that provides the relative concentrations of water and secondary phases of the mineral assemblage in the microinclusions (i.e., carbonates, silicates and apatite) that grew from trapped HDFs. Constraining the relative abundances of these phases provides information on the amount of water and carbonate in different HDF types, and was proven as a semi-quantitative method to determine carbonatitic to silicic compositions (Weiss et al., 2010). However this was accomplished by manual subtraction of diamond intrinsic absorption and nitrogen absorption, followed by deconvolution of the IR absorbance due to various phases in the micro-inclusions; a long and slow process to be conducted routinely in the study of fluid-rich diamonds.

The complete automation of deconvoluting fluid inclusion-rich diamond IR spectra is complex for the following reasons: (1) a 'one size fits all' approach to baselining and subtracting the various components in the spectrum is almost impossible given the chemical variability observed; (2) automation offers little to no user refinement, and the need to discern the quality of the data output undermines the time saved by automating it. We therefore developed a semi-automated approach DiaMap_Fluid - for fast subtraction and deconvolution processing of a fluid inclusion-rich diamond IR spectrum. This software allows for user refinement during each stage of spectral processing, 
making it a much faster user process, while also greatly enhancing precision in the data output from a single spectrum compared with manual processing by different individuals.

We have applied the DiaMap_Fluid program to 38 fluid inclusion-rich diamonds, from localities in Africa (Finsch, Kankan, DeBeers-Pool, Koingnass), Canada (Ekati) and Russia (Udachnaya, Aykhal). The samples have been studied by quantitative methods including electron microprobe and laser ablation ICPMS (Weiss et al., 2008; 2010), allowing the FTIR data on the water (OH), carbonate and silicate components of the microinclusions to be compared with their bulk chemistry. The results provide the phase concentrations (in ppm by weight) in the diamonds, and show that on a ternary plot of carbonate - water - silicates + apatite (Figure 1), the four HDF end-members fall in fairly distinct groupings. This makes FTIR analyses using the DiaMap_Fluid software a very simple method for determining phase concentations and broadly discerning the chemistry of HDF in fluid-rich diamonds.

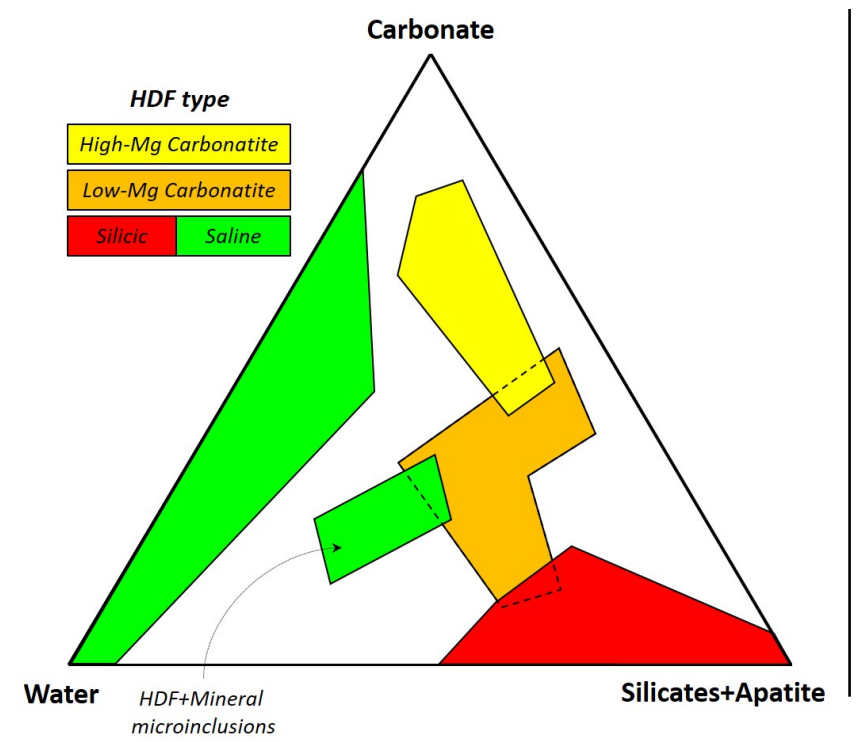

Figure 1: Carbonate - water - silicates + apatite ternary plot showing the relative amount of daughter phases in microinclusions of different HDF types. The fields for HDF in 38 diamonds (high-Mg carbonatite - 13; low-Mg carbonatite 5; saline - 11; silicic - 9) show minimal overlap, allowing easy and fast characteristics of HDF type in diamonds using the IR analyses and the DiaMap_Fluid software. The small saline field plots away from the water - carbonate axis due to a large proportion of micro-minerals relative to HDF micro-inclusions, in 2 diamonds studied.

\section{DiaMap_Type_Ib}

The mapping of isolated nitrogen $\left(\mathrm{N}_{\mathrm{S}}\right)$ in any natural or synthetic diamond is a relatively simple process compared to the complexities of mantle fluids, and as such has been feasible for some time (Babich \& Fiegelson, 2009). Isolated nitrogen produces vibrational peaks at both $1344 \mathrm{~cm}^{-1}$ and 1130 $\mathrm{cm}^{-1}$ that are correlated with each other. Since the $1344 \mathrm{~cm}^{-1}$ peak is sharper, with a clearer peakshape to fit at low concentrations compared to the broad $1130 \mathrm{~cm}^{-1}$ peak, we decided to employ this peak to calculate $\mathrm{N}_{\mathrm{S}}$ in DiaMap_Ib. Given the two peaks are correlated, data can still be compared between our new datasets and previous datasets that employ the $1130 \mathrm{~cm}^{-1}$ calibration.

DiaMap_Ib was used to map $\mathrm{N}_{\mathrm{S}}$ in a suite of 15 natural Neoproterozoic Ib diamonds from the Zimmi alluvial deposits in West Africa (Smit et al., 2016). IR absorption was analysed at $100 \mu \mathrm{m}$ intervals across each double-sided plate, with a high spectral resolution of $1 \mathrm{~cm}^{-1}$ to resolve the sharp $1344 \mathrm{~cm}^{-1}$ peak. In the 15 samples that were spectroscopically analysed, $\mathrm{N}_{\mathrm{S}}$ averages between 12 and $54 \mathrm{ppm}$, though areas of higher $\mathrm{N}_{\mathrm{S}}$ (up to $150 \mathrm{ppm}$ ) are observed in the FTIR maps. Comparison between DiaMap_Ib output with N concentrations measured by SIMS show excellent agreement. Fancy brownish-yellow to greenish-yellow colours observed in Zimmi Ib diamonds result mainly from $\mathrm{N}_{\mathrm{S}}$, although deformation-related defects may also contribute to body colour. Maps of $\mathrm{N}_{\mathrm{S}}$ concentration in these diamonds are being used to better understand the relationship between $\mathrm{N}_{\mathrm{S}}$ and colour distribution, since previous studies have suggested no direct correlation. In the suite as a whole, average $\mathrm{N}_{\mathrm{S}}$ (calculated from all the spectra in the FTIR maps) does not correlate to the most vivid colours. For individual diamonds, FTIR mapping will help us understand how $\mathrm{N}_{\mathrm{S}}$ concentration affects colour concentration between core and rim, and how $\mathrm{N}_{\mathrm{S}}$ impacts the formation of other defects (e.g., NV centres) during exhumation-related deformation (Smit et al., 2016). 


\section{DiaMap_Type_IIb}

The study of boron in natural diamonds has been very limited due to their high value. Uncompensated boron (i.e., the amount of boron that is in excess of nitrogen) is IR active and can produce several features that have been calibrated for their concentration (Collins, 2010). The primary B peak is $\sim 2800 \mathrm{~cm}^{-1}\left(0.035 \pm 0.0028 \times \mathrm{H}_{2800} \mathrm{ppm}\right)$ with a secondary feature at $\sim 2458 \mathrm{~cm}^{-1}(0.105 \pm 0.008 \times$ $\mathrm{H}_{2458} \mathrm{ppm}$ ). Boron concentration in natural diamonds is commonly $<1 \mathrm{ppm}$, however in synthetic diamonds, B concentrations can be orders of magnitude higher ( $>50 \mathrm{ppm}$; Collins, 2010). As B concentration increases, the $2800 \mathrm{~cm}-1$ peak broadens significantly and is no longer quantifiable, but an additional peak appears in the one phonon region $\sim 1290 \mathrm{~cm}^{-1}\left(1.00 \pm 0.15 \times \mathrm{H}_{1290} \mathrm{ppm}\right.$; Collins, 2010). As broadening of the primary peak occurs, normalising and subtraction of a Type IIa spectrum by the usual fitting procedure becomes impossible and can only be achieved using calculations involving a sample's thickness. FTIR mapping of B concentration in Type IIb diamonds is important for understanding growth sector dependencies for B incorporation.

Since Type IIb mapping has never previously been achieved, we developed DiaMap_IIb. This routine utilises either the $2800 \mathrm{~cm}^{-1}$ or $1290 \mathrm{~cm}^{-1}$ peak, depending on whether the primary peak is saturated or not. Mapping of two synthetic samples (provided by New Diamond Technologies) has shown that B is preferentially incorporated into the $\{111\}$ growth sectors, relative to the $\{100\}$ sectors, with an enrichment factor of $>30(\sim 0.5 \mathrm{ppm}$ in $\{100\} \mathrm{vs} \sim 17 \mathrm{ppm}$ in $\{111\}$; Figure 2$)$. In addition to the large inter-sectorial variations, more subtle intra-sectorial variations exist within the $\{100\}$ sectors. This suggests additional factors affect impurity uptake beyond just the difference in bonding opportunities on different crystal faces.
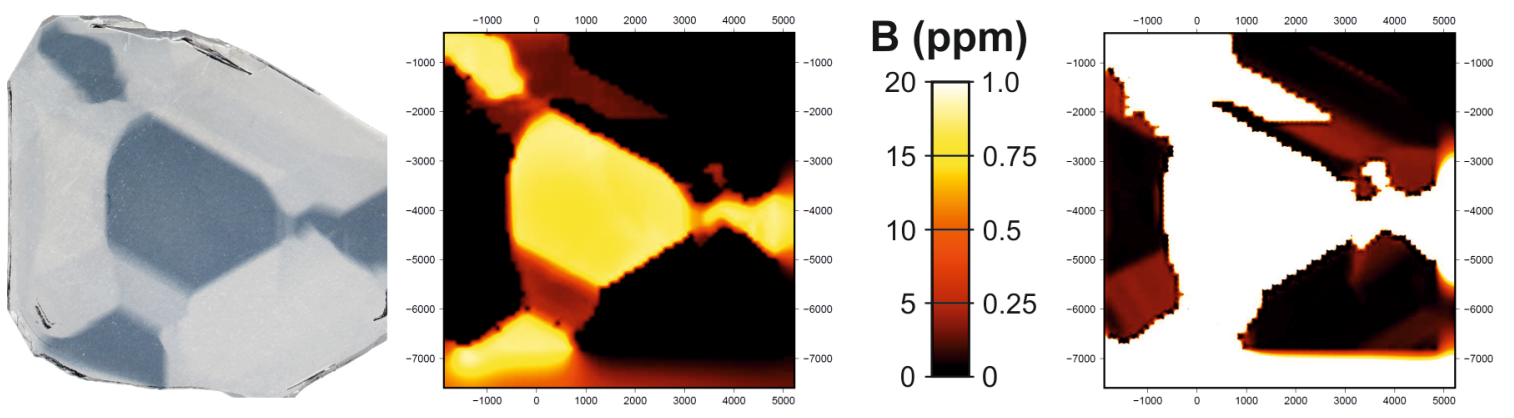

Figure 2: A regular light photograph of a synthetic Type IIb diamond, along with two maps showing boron concentrations (in ppm) generated from the FTIR mapping. The difference between the two maps is the scale bar. In the map on the left, the colour scale runs from $0-20 \mathrm{ppm}$, showing the large concentration difference between growth sectors. In the map on the right, the colour scale only runs from $0-1 \mathrm{ppm}$, highlighting subtle variations within the low concentration $\{100\}$ sectors, while the $\{111\}$ sectors are just shown in white.

\section{References}

Babich YV, Fiegelson BN, 2009. Spatial distribution of the nitrogen defects in synthetic diamond monocrystals: data of IR mapping. Geochemistry International, 47, 94.

Collins A, 2010. Determination of the boron concentration in diamond using optical spectroscopy. Proceedings $61^{\text {st }}$ Diamond Conference, Warwick.

Howell D, O’Neill CJ, Grant KJ, Griffin WL, Pearson NJ, O'Reilly SY 2012. FTIR mapping: distribution of impurities in different types of diamond growth. Diamond \& Related Materials, 29, 29.

Navon O, Hutcheon ID, Rossman GR, Wasserburg GJ, 1988. Nature, 335, 784.

Robertson R, Fox JJ, Martin AE, 1934. Two types of diamond. Phil. Trans. Roy. Soc. Lond., 232, 463.

Smit KV, Shirey SB, Wang W, 2016. Type Ib diamond formation and preservation in the West African lithospheric mantle: Re-Os age constraints. Precambrian Research, 286, 152.

Weiss Y, Kiflawi I, Navon O, 2010. IR spectroscopy: quantitative determination of the mineralogy \& bulk composition of fluid microinclusions in diamonds. Chemical Geology, 275, 26.

Weiss Y, Griffin WL, Elhlou S, Navon O, 2008. Comparison between LA-ICP-MS and EMPA analysis of trace elements in diamonds. Chemical Geology, 252, 158. 